\title{
Spreading dynamics of a preferential information model with hesitation psychology on scale-free networks
}

\author{
Xiongding Liu ${ }^{1,2}$, Tao Li ${ }^{1,2^{*}}$, Xinming Cheng ${ }^{3}$, Wenjin Liu ${ }^{1,2}$ and Hao X $\mathrm{u}^{1,2}$
}

\author{
"Correspondence: \\ taohust2008@163.com \\ ${ }^{1}$ National Demonstration Center for \\ Experimental Electrical and \\ Electronic Education, Yangtze \\ University, Jingzhou, P.R. China \\ ${ }^{2}$ School of Electronics and \\ Information, Yangtze University, \\ Jingzhou, P.R. China \\ Full list of author information is \\ available at the end of the article
}

\begin{abstract}
Considering the influence of the preferential degree, the hesitation psychology of customers and the heterogeneity of underlying networks in preferential information spreading, we propose a novel model called AHFB (adherent-hesitator-forwarderbeneficiary) model to illustrate the dynamic behaviors of preferential information spreading on scale-free networks. The mean-field theory is adopted to describe the formulas of AHFB model. To begin with, we analyze the spreading dynamics of preferential information. Then we determine the basic reproductive number and equilibria by the next generation matrix method. The relationship among the basic reproductive number, preferential degree, and hesitation parameter is also analyzed. In addition, the globally asymptotical stability of information-eliminate equilibrium and the permanence of the preferential information spreading are proved in detail. Furthermore, a preferential information competition model is proposed, and the corresponding dynamic behaviors are studied. We have found that under certain conditions, a greater competitive advantage can be achieved by improving a certain range of preferential strengths. Numerical simulations are also presented to verify and extend theoretical results.
\end{abstract}

Keywords: Attractivity parameter; Preferential information spread model; Heterogeneity; Scale-free networks; Hesitation psychology

\section{Introduction}

With the continuous prosperity of mobile Internet technology and the appearance of the 5G, e-commerce technology is rapidly developing and multifarious applications are emerging. As a kind of Internet networks, e-commerce networks not only play an important role in spreading information and promoting communication, but also penetrate people's daily life, such as convenient shopping, mobile payment [1-3]. In most instances, e-commerce networks provide businesses with a platform to release preferential information and display merchandises $[4,5]$. In order to obtain discounts or small gifts from the store during the shopping process, customers need to forward the corresponding preferential information [6]. Therefore, the preferential information will be spread widely.

In the field of complex networks, researchers have made some achievements in information spreading [7-14] and network control [15-19]. Based on the characteristics of network structure, the effects of different network topologies, such as small world net-

(c) The Author(s) 2019. This article is distributed under the terms of the Creative Commons Attribution 4.0 International License (http://creativecommons.org/licenses/by/4.0/), which permits unrestricted use, distribution, and reproduction in any medium, provided you give appropriate credit to the original author(s) and the source, provide a link to the Creative Commons license, and indicate if changes were made. 
works, random networks, scale-free networks, community structure networks, and multiplex networks, on information spreading models are studied in detail. The authors of [20] described information spreading by ODE (ordinary differential equation) dynamic system and built a linear algebra model in small world networks. They studied the relationship between the ODE function groups and the graph topology, and also found the rule of the information's distribution in networks. Lim et al. [21] studied the influence of clustering coefficients under the SIR model with heterogeneous contact rates and provided a novel iterative algorithm to estimate the conditions and sizes of global cascades. In order to study the influence of human subjective value and psychological status on information dissemination, Liu et al. [22] proposed a novel social network information dissemination with negative feedback NFSIR (negative feedback-susceptible-infected-removed) model on scale-free networks, which showed that the intensity of information feedback has a significant impact on the process of information dissemination. The authors of [23, 24] presented a privacy protection and emotional behavior information spreading model with a community structure on social networks. Zhang et al. $[25,26]$ proposed a node measurement augmented system model in multirate systems networks with dynamic quantization. Recently, scholars have begun to take serious consideration about the role of human behavior and the multiplex networks structure in information spreading [27-29]. The interplay between the epidemic spreading and the diffusion of awareness in multiplex networks was analyzed in [30, 31].

Another generalization of the initial simple determinacy is to focus on different models and spreading mechanisms. The typical information behavior in social networks is a kind of information sharing and interaction. Ally et al. [32] proposed two rewiring SIR (susceptible-infected-removed) models on information spreading in scale-free and small world networks. In order to study the effect of hesitation mechanism, the authors of [33,34] proposed an SEIR (susceptible-exposed-infected-removed) model by introducing the exposed nodes between the ignorant nodes and the spread nodes in heterogeneity networks, they found that hesitant individuals have a very important influence on the spreading of information. To further investigate the influence of heterogeneity of the underlying complex networks and quarantine strategy, an SIQRS (susceptible-infectedquarantined-recovered-susceptible) epidemic model on the scale-free networks has been proposed $[35,36]$.

It has been found that most real networks are actually scale-free networks, so the scalefree property is a particularly important one in social networks [37, 38]. To further understand the information spreading dynamics in real world, the scale-free property of social networks has been taken into account by many information spreading models [39-41]. In networks, nodes represent individual and edges represent the relationship of people, as for preferential spreading dynamics, that relationship is contacted to forward the preferential information. With the rapid development of social networks and new media, increasingly many business groups choose social networks to promote their products by publishing preferential information. Hence, it is of great importance to study the spreading dynamics of preferential information on scale-free networks. Due to the influences of the merchandise's preferential degree, personal economic level, psychological and other factors, some customers will not immediately forward the preferential information after they know it in the real social life, and they will become hesitant at first. However, in the literature on preferential information spreading, some researchers ignore the hesitating mechanism, 
which will affect people's attitudes and behaviors on preferential information and further influence its spreading. People in the hesitating state will read some comments or opinions about the products because of the attractiveness of preferential information, which may make one enter the forwarder status to forward the preferential information with a certain probability. An FSFC (follower-super-forwarder-client) preferential information model was proposed by Fu et al. [42] in online social networks, who found that different infection rates have a great impact on large- and small-degree node forwarders. Wan et al. [43] proposed an SIB (susceptible-infected-beneficial) model based on scale-free networks, and they further studied the influence of the preferential degree and the heterogeneity of underlying networks on the spread of preferential e-commerce information. However, the influence of the parameters in the model on the basic reproductive number has not been analyzed in detail, and the hesitation psychology of people is also not considered. Motivated by the above, we establish a novel $A H F B$ preferential information spreading model with hesitation mechanism on scale-free networks.

The remainder of this paper is organized as follows. Section 2 presents an AHFB preferential information spreading model. In Sect. 3, the basic reproductive number and equilibriums are obtained. Section 4 analyzes the globally asymptotic stability of equilibriums and the permanence of preferential information spreading. In Sect. 5, the modified preferential information spreading model with competitive mechanism is introduced and the corresponding dynamical behaviors are studied. In Sect. 6, numerical simulations are presented to illustrate our main results. Finally, we give the discussions and conclusions in Sect. 7.

\section{Model formulation}

During preferential information spreading in a population, individuals are divided into four categories: adherents, hesitators, forwarders, and beneficiaries. Adherents are individuals who never know the preferential information. Hesitators are individuals who know the preferential information in hesitation state and do not forward preferential information. Forwarders are individuals who know and forward the preferential information. Beneficiaries are individuals who know the preferential information and obtain the preferential policies. Taking the heterogeneity induced by the presence of nodes with different connectivity into account, we let $A_{k}(t), H_{k}(t), F_{k}(t)$, and $B_{k}(t)$ be the relative densities of adherent, hesitator, forwarder and beneficiary nodes of degree $k$ at time $t$, respectively. Figure 1 illustrates the spreading rules of the $A H F B$ model. We assume that new immigration individuals are adherent at rate $b(k)$, the emigration rate of all individuals is $\mu$. The reconnection of these new immigration nodes follows the below propagation rules. If an adherent is connected to a forwarder, then he or she will be infected to become a hesitator or a forwarder with a probability of $\delta \rho_{1}$ or $\alpha \rho_{1}$, respectively. If an adherent is connected to a beneficiary, then he or she will be infected to become a hesitator or a forwarder with a probability of $\delta \rho_{2}$ or $\alpha \rho_{2}$, respectively. Here, the parameter $\delta$ is used to describe the hesitation degree of adherents, the attractivity parameter $\alpha$ is used to describe the preferential degree of preferential information. A hesitator becomes a forwarder with a probability of $\beta$. Parameter $\rho_{1}\left(\rho_{2}\right)$ is the spread rate for an adherent after contacting with a forwarder (beneficiary). A forwarder becomes a beneficiary with a probability of $\gamma$ when he or she forwards the preferential information a specific number of times. 


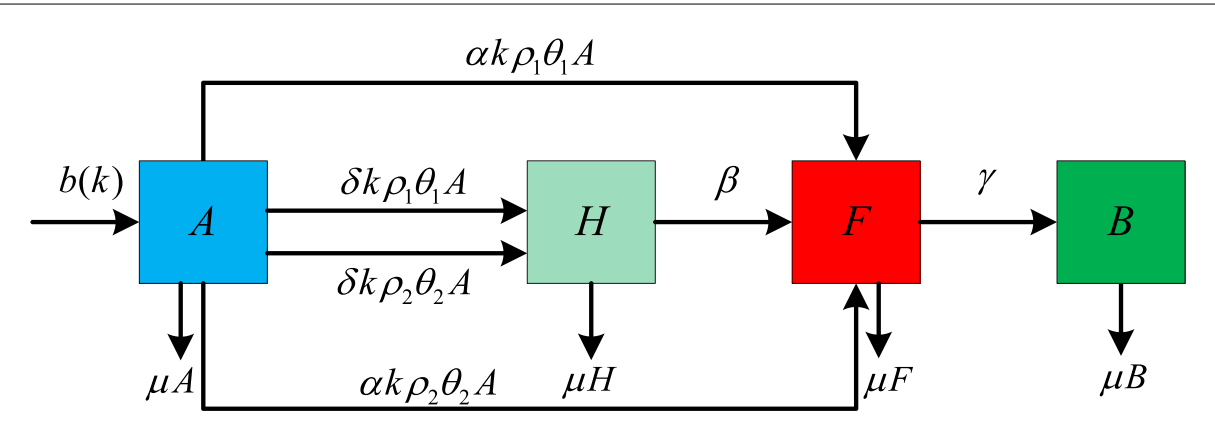

Figure 1 The flow diagram of the AHFB model

Based on the above assumptions and conditions of hypothesis, the AHFB preferential information spreading dynamics model can be described by

$$
\left\{\begin{aligned}
\frac{d A_{k}(t)}{d t}= & b(k)-\alpha k\left(\rho_{1} \theta_{1}(t)+\rho_{2} \theta_{2}(t)\right) A_{k}(t)-\delta k\left(\rho_{1} \theta_{1}(t)\right. \\
& \left.+\rho_{2} \theta_{2}(t)\right) A_{k}(t)-\mu A_{k}(t), \\
\frac{d H_{k}(t)}{d t}= & \delta k\left(\rho_{1} \theta_{1}(t)+\rho_{2} \theta_{2}(t)\right) A_{k}(t)-\beta H_{k}(t)-\mu H_{k}(t), \\
\frac{d F_{k}(t)}{d t}= & \alpha k\left(\rho_{1} \theta_{1}(t)+\rho_{2} \theta_{2}(t)\right) A_{k}(t)+\beta H_{k}(t)-\gamma F_{k}(t)-\mu F_{k}(t), \\
\frac{d B_{k}(t)}{d t}= & \gamma F_{k}(t)-\mu B_{k}(t),
\end{aligned}\right.
$$

where $\theta_{1}(t), \theta_{2}(t)$ denote the probabilities of an adherent contacts with a forwarder or a beneficiary at time $t$, respectively, which satisfies the relations

$$
\left\{\begin{array}{l}
\theta_{1}(t)=\sum_{i=1}^{n} \frac{\varphi(i)}{i} P(i \mid k) \frac{F_{i}(t)}{N_{i}(t)}=\langle k\rangle^{-1} \sum_{i=1}^{n} \frac{\varphi(i)}{\eta_{i}} P(i) F_{i}(t), \\
\theta_{2}(t)=\sum_{i=1}^{n} \frac{\varphi(i)}{i} P(i \mid k) \frac{B_{i}(t)}{N_{i}(t)}=\langle k\rangle^{-1} \sum_{i=1}^{n} \frac{\varphi(i)}{\eta_{i}} P(i) B_{i}(t) .
\end{array}\right.
$$

Here, the factor $1 / i$ stands for the probability that one of the forwarder or beneficiary neighbors of a node with degree $i$ will contact this node at the present time step; $P(i \mid k)$ denotes the conditional probability that a node with degree $k$ is connected to a node with degree $i$. In this paper, $P(i \mid k)=i P(k) /\langle k\rangle$ and $\sum_{k=1}^{n} P(k)=1 ;\langle k\rangle=\sum_{k=1}^{n} k P(k)$ denotes the average degree. The function $\varphi(t)$ is the forwarder node with degree $k$, and $\rho(t)=\rho_{1} \theta_{1}(t)+$ $\rho_{2} \theta_{2}(t)$. And $N_{k}(t)$ stands for the total number of nodes with degree $k$ at time $t$, which satisfies

$$
N_{k}(t)=A_{k}(t)+H_{k}(t)+F_{k}(t)+B_{k}(t)
$$

It is easy to see that $d N_{k}(t) / t=b(k)-\mu N_{k}(t)$, so $N_{k}(t)=\left(N_{0}-b(k) / \mu\right) e^{-u t}+b(k) / \mu$, where $N_{0}=N_{k}(0)$, and then $\lim _{x \rightarrow \infty} N_{k}(t)=b(k) / \mu=\eta_{k}$. Thus, since system (2.1) monitors human population, it is reasonable to assume that all its state variables and parameters are nonnegative for all $t \geq 0$. It implies that the region

$$
\begin{aligned}
\Omega= & \left\{A_{k}(t), H_{k}(t), F_{k}(t), B_{k}(t) \in R_{+}^{4 n} \mid A_{k}(t)+H_{k}(t)+F_{k}(t)+B_{k}(t) \leq \eta_{k},\right. \\
& k=1,2, \ldots, n\}
\end{aligned}
$$


is the positively invariant set for (2.1). Thus, each solution of system (2.1) with initial conditions and the limit sets are contained in $\Omega$ in this paper.

Since $A_{k}(t)=\eta_{k}-H_{k}(t)-F_{k}(t)-B_{k}(t)$ is at steady-state, it is sufficient to study the limiting system

$$
\left\{\begin{aligned}
\frac{d H_{k}(t)}{d t}= & \delta k\left(\rho_{1} \theta_{1}(t)+\rho_{2} \theta_{2}(t)\right)\left(\eta_{k}-H_{k}(t)-F_{k}(t)-B_{k}(t)\right) \\
& -\beta H_{k}(t)-\mu H_{k}(t) \\
\frac{d F_{k}(t)}{d t}= & \alpha k\left(\rho_{1} \theta_{1}(t)+\rho_{2} \theta_{2}(t)\right)\left(\eta_{k}-H_{k}(t)-F_{k}(t)-B_{k}(t)\right) \\
& +\beta H_{k}(t)-\gamma F_{k}(t)-\mu F_{k}(t) \\
\frac{d B_{k}(t)}{d t}= & \gamma F_{k}(t)-\mu B_{k}(t) .
\end{aligned}\right.
$$

\section{The basic reproductive number and existence of equilibriums}

\subsection{The basic reproductive number $\boldsymbol{R}_{\mathbf{0}}$}

System (2.5) has an information-elimination equilibrium $E_{0}\{(0,0,0)\}_{k}$. Using the next generation method in [44], system (2.5) can be written as

$$
\frac{d x}{d t}=M(x)-V(x)
$$

where

$$
\begin{aligned}
& x=\left(H_{k}, F_{k}, B_{k}\right)^{T}, \\
& M(x)=\left(\begin{array}{c}
\delta k \rho(t)\left(\eta_{k}-H_{k}-F_{k}-B_{k}\right) \\
\alpha k \rho(t)\left(\eta_{k}-H_{k}-F_{k}-B_{k}\right) \\
0
\end{array}\right), \\
& V(x)=\left(\begin{array}{c}
(\beta+\mu) H_{k} \\
-\beta H_{k}+(\gamma+\mu) F_{k} \\
\mu B_{k}-\gamma F_{k}
\end{array}\right) .
\end{aligned}
$$

The Jacobian matrices of $M(x)$ and $V(x)$ at the information-elimination equilibrium $E_{0}$ are as follows:

$$
M(x)=D M\left(E_{0}\right)=\left(\begin{array}{ccc}
0 & M_{12} & M_{13} \\
0 & M_{22} & M_{23} \\
0 & 0 & 0
\end{array}\right), \quad V(x)=D V\left(E_{0}\right)=\left(\begin{array}{ccc}
V_{11} & 0 & 0 \\
V_{21} & V_{22} & 0 \\
0 & V_{32} & V_{33}
\end{array}\right),
$$

where

$$
M_{12}=\frac{\delta \eta_{k} \rho_{1}}{\langle k\rangle}\left(\begin{array}{cccc}
\frac{\varphi(1)}{\eta_{1}} P(1) & \frac{\varphi(2)}{\eta_{1}} P(2) & \ldots & \frac{\varphi(n)}{\eta_{1}} P(n) \\
\frac{\varphi(1)}{\eta_{2}} 2 P(1) & \frac{\varphi(2)}{\eta_{2}} 2 P(2) & \ldots & \frac{\varphi(n)}{\eta_{2}} 2 P(n) \\
\vdots & \vdots & \ddots & \vdots \\
\frac{\varphi(1)}{\eta_{n}} n P(1) & \frac{\varphi(2)}{\eta_{n}} 2 n P(2) & \ldots & \frac{\varphi(n)}{\eta_{n}} n P(n)
\end{array}\right),
$$




$$
\begin{gathered}
M_{13}=\frac{\delta \eta_{k} \rho_{2}}{\langle k\rangle}\left(\begin{array}{cccc}
\frac{\varphi(1)}{\eta_{1}} P(1) & \frac{\varphi(2)}{\eta_{1}} P(2) & \ldots & \frac{\varphi(n)}{\eta_{1}} P(n) \\
\frac{\varphi(1)}{\eta_{2}} 2 P(1) & \frac{\varphi(2)}{\eta_{2}} 2 P(2) & \ldots & \frac{\varphi(n)}{\eta_{2}} 2 P(n) \\
\vdots & \vdots & \ddots & \vdots \\
\frac{\varphi(1)}{\eta_{n}} n P(1) & \frac{\varphi(2)}{\eta_{n}} 2 n P(2) & \ldots & \frac{\varphi(n)}{\eta_{n}} n P(n)
\end{array}\right), \\
M_{22}=\frac{\alpha \eta_{k} \rho_{1}}{\langle k\rangle}\left(\begin{array}{cccc}
\frac{\varphi(1)}{\eta_{1}} P(1) & \frac{\varphi(2)}{\eta_{1}} P(2) & \ldots & \frac{\varphi(n)}{\eta_{1}} P(n) \\
\frac{\varphi(1)}{\eta_{2}} 2 P(1) & \frac{\varphi(2)}{\eta_{2}} 2 P(2) & \ldots & \frac{\varphi(n)}{\eta_{2}} 2 P(n) \\
\vdots & \vdots & \ddots & \vdots \\
\frac{\varphi(1)}{\eta_{n}} n P(1) & \frac{\varphi(2)}{\eta_{n}} 2 n P(2) & \ldots & \frac{\varphi(n)}{\eta_{n}} n P(n)
\end{array}\right), \\
M_{23}=\frac{\alpha \eta_{k} \rho_{2}}{\langle k\rangle}\left(\begin{array}{cccc}
\frac{\varphi(1)}{\eta_{1}} P(1) & \frac{\varphi(2)}{\eta_{1}} P(2) & \ldots & \frac{\varphi(n)}{\eta_{1}} P(n) \\
\frac{\varphi(1)}{\eta_{2}} 2 P(1) & \frac{\varphi(2)}{\eta_{2}} 2 P(2) & \ldots & \frac{\varphi(n)}{\eta_{2}} 2 P(n) \\
\vdots & \vdots & \ddots & \vdots \\
\frac{\varphi(1)}{\eta_{n}} n P(1) & \frac{\varphi(2)}{\eta_{n}} 2 n P(2) & \ldots & \frac{\varphi(n)}{\eta_{n}} n P(n)
\end{array}\right),
\end{gathered}
$$

here $V_{11}=(\beta+\mu) I, V_{21}=-\beta I, V_{22}=(\gamma+\mu) I, V_{32}=-\gamma I, V_{33}=\mu I, I$ is the identity matrix, and $\mathbf{0}$ is the zero matrix. The basic reproductive number is denoted by

$$
R_{0}=\rho\left(M V^{-1}\right)=\frac{\langle\varphi(k) k\rangle\left(\mu \rho_{1}+\gamma \rho_{2}\right)(\alpha(\mu+\beta)+\beta \delta)}{\langle k\rangle \mu(\gamma+\mu)(\beta+\mu)},
$$

where $\langle\varphi(k) k\rangle=\sum_{i=1} k \varphi(k) P(k), i=1,2, \ldots, n$.

\subsection{Existence of equilibriums}

Theorem 1 Consider system (2.1) and define $R_{0}=\frac{\langle\varphi(k) k\rangle}{\langle k\rangle} \frac{(\alpha(\mu+\beta)+\delta \beta)\left(\mu \rho_{1}+\gamma \rho_{2}\right)}{\mu(\beta+\mu)(\mu+\gamma)}$. There always exists an information-elimination equilibrium $E_{0}\left(\eta_{k}, 0,0,0\right)$ when $R_{0}<1$. When $R_{0}>1$, the system has an information-prevailing equilibrium $E^{*}\left(A_{k}^{*}, H_{k}^{*}, F_{k}^{*}, B_{k}^{*}\right)$.

Proof To get the information-prevailing equilibrium solution $E^{*}\left(A_{k}^{*}, H_{k}^{*}, F_{k}^{*}, B_{k}^{*}\right)$, we need to make the right-hand side of the system equal to zero. It should satisfy

$$
\left\{\begin{array}{l}
b(k)-\alpha k A_{k}^{*}(t)\left(\rho_{1} \theta_{1}^{*}(t)+\rho_{2} \theta_{2}^{*}(t)\right)-\delta k A_{k}^{*}(t)\left(\rho_{1} \theta_{1}^{*}(t)+\rho_{2} \theta_{2}^{*}(t)\right)-\mu A_{k}^{*}(t)=0 \\
\delta k A_{k}^{*}(t)\left(\rho_{1} \theta_{1}^{*}(t)+\rho_{2} \theta_{2}^{*}(t)\right)-\beta H_{k}^{*}(t)-\mu H_{k}^{*}(t)=0 \\
\alpha k A_{k}^{*}(t)\left(\rho_{1} \theta_{1}^{*}(t)+\rho_{2} \theta_{2}^{*}(t)\right)+\beta H_{k}^{*}(t)-\gamma F_{k}^{*}(t)-\mu F_{k}^{*}(t)=0 \\
\gamma F_{k}^{*}(t)-\mu B_{k}^{*}(t)=0
\end{array}\right.
$$

where $\theta_{1}^{*}=\langle k\rangle^{-1} \sum_{i=1}^{n} \frac{\varphi(k)}{\eta_{k}} P(k) F_{k}^{*}(t), \theta_{2}^{*}=\langle k\rangle^{-1} \sum_{i=1}^{n} \frac{\varphi(k)}{\eta_{k}} P(k) B_{k}^{*}(t)$. We obtain from (3.6) that

$$
\begin{aligned}
A^{*} & =\frac{(\beta+\mu)(\gamma+\mu)}{k \rho^{*}(\alpha(\beta+\mu)+\beta \delta)} F^{*}, \\
H^{*} & =\frac{\delta(\gamma+\mu)}{\alpha(\beta+\mu)+\beta \delta} F^{*}, \\
B^{*} & =\frac{\gamma}{\mu} F^{*},
\end{aligned}
$$


where $\rho=\rho_{1} \theta_{1}+\rho_{2} \theta_{2}$. From (3.7) we get $\theta_{2}^{*}=\gamma \theta_{1}^{*} / \mu$. Considering the normalization condition, we obtain

$$
F^{*}=\frac{\mu k \rho^{*}(\alpha(\beta+\mu)+\delta \beta) \eta_{k}}{\left(k \rho^{*}(\delta+\alpha)+\mu\right)(\gamma+\mu)(\beta+\mu)} .
$$

Inserting it into (2.2), we obtain that

$$
\theta_{1}^{*}=\frac{1}{\langle k\rangle} \sum_{i=1}^{n} \frac{\varphi(k)}{\eta_{k}} P(k) \frac{k\left(\mu \rho_{1}+\gamma \rho_{2}\right)(\alpha(\beta+\mu)+\delta \beta) \eta_{k}}{\left(k \rho^{*}(\delta+\alpha)+\mu\right)(\beta+\mu)(\gamma+\mu)} \theta_{1}^{*}=f\left(\theta_{1}^{*}\right) .
$$

Clearly, $\theta_{1}^{*}=0$ satisfies above equation, and if $\theta_{1}^{*}=0$, then $\theta_{2}^{*}=0$ and $H_{k}^{*}=F_{k}^{*}=B_{k}^{*}=0$, which is an information-elimination equilibrium of (2.1). It is easy to verify that $f\left(\theta_{1}^{*}\right)$ is a convex and increasing function when $\left.f\left(\theta_{1}^{*}\right)\right|_{\theta_{1}^{*}=1}<1$. So if $\theta_{1}^{*}$ has an another solution in $[0$, 1], it must satisfy

$$
\left.\frac{d f\left(\theta_{1}^{*}\right)}{d \theta_{1}^{*}}\right|_{\theta_{1}^{*}=0}=\frac{\langle\varphi(k) k\rangle}{\langle k\rangle} \frac{(\alpha(\mu+\beta)+\delta \beta)\left(\mu \rho_{1}+\gamma \rho_{2}\right)}{\mu(\beta+\mu)(\mu+\gamma)}=R_{0}>1,
$$

and we can obtain the basic reproductive number

$$
R_{0}=\frac{\langle\varphi(k) k\rangle}{\langle k\rangle} \frac{(\alpha(\mu+\beta)+\delta \beta)\left(\mu \rho_{1}+\gamma \rho_{2}\right)}{\mu(\beta+\mu)(\mu+\gamma)}>1 .
$$

So, a nontrivial solution exists if and only if $R_{0}>1$. Inserting the nontrivial solution of (3.7) into Eq. (3.6), we obtain $F_{k}^{*}$. Then by (3.7) and (3.8), we can easily get $0<A_{k}^{*}, H_{k}^{*}, F_{k}^{*}, B_{k}^{*}<\eta_{k}$ for $k=1,2, \ldots, n$. Thus, the equilibrium $E^{*}\left(A_{k}^{*}, H_{k}^{*}, F_{k}^{*}, B_{k}^{*}\right)$ is welldefined. Hence, when $R_{0}>1$, only one positive equilibrium $E^{*}\left(A_{k}^{*}, H_{k}^{*}, F_{k}^{*}, B_{k}^{*}\right)$ of system (2.1) exists. The proof is completed.

\section{Remark}

(1) The basic reproductive number $R_{0}$ depends on some model parameters and fluctuations of the degree distribution. It can be found that $R_{0}$ has no correlation with the degree-dependent new immigration individuals $b(k)$. It seems that the attraction parameters $\alpha$ and the infection rate $\rho_{1}, \rho_{2}$ have the same effects, because $R_{0}$ will increase when they increase, the effects will be explored by the detailed numerical calculation.

(2) If $\varphi(k)=k, \beta=0$, and $b(k)=\mu$ (i.e., new immigration individuals are balanced by emigration rate), then the model can be simplified to the network based SIB model with $R_{0}=\frac{\left\langle k^{2}\right\rangle}{\langle k\rangle} \frac{v\left(l \beta_{1}+\varepsilon \beta_{2}\right)}{l(l+\varepsilon)}$, which is investigated in [43].

\section{Global dynamics of the model}

In this section, qualitative analysis of the model is presented. Firstly, we consider the local asymptotical stability of the information-elimination equilibrium $E_{0}$.

Theorem 2 For system (2.5), the information-elimination equilibrium $E_{0}$ is locally asymptotically stable if $R_{0}<1$. 
Proof For the purpose of using the comparison theorem to prove the global stability of the information-elimination equilibrium, we assume that $H_{1}=z_{1}, H_{2}=z_{2}, \ldots, H_{n}=z_{n}, F_{1}=$ $z_{n+1}, F_{2}=z_{n+2}, \ldots, F_{n}=z_{2 n}, B_{1}=z_{2 n+1}, B_{2}=z_{2 n+2}, \ldots, B_{n}=z_{3 n}$, and $z=\left(z_{1}, \ldots, z_{n}, z_{n+1}, \ldots\right.$, $\left.z_{2 n}, z_{2 n+1}, \ldots, z_{3 n}\right)^{T}$, then system (2.5) can be written as

$$
\frac{d z}{d t}=A z+N(z)
$$

where

$$
A=M-V, \quad N(z)=-\left(\begin{array}{c}
\rho\left(z_{1}+z_{n+1}+z_{2 n+1}\right) \\
2 \rho\left(z_{2}+z_{n+2}+z_{2 n+2}\right) \\
\vdots \\
n \rho\left(z_{n}+z_{2 n}+z_{3 n}\right) \\
(n+1) \rho\left(z_{n}+z_{2 n}+z_{3 n}\right) \\
\vdots \\
2 n \rho\left(z_{n}+z_{2 n}+z_{3 n}\right) \\
0 \\
\vdots \\
0
\end{array}\right)_{3 n \times 1}
$$

the matrices $M$ and $V$ satisfy (3.1); and, as one can see above, $N(z)$ is negative. So $\frac{d z}{d t} \leq$ $A z$. We can find that the off-diagonal elements of matrix $A$ are nonnegative, hence, the quasimonotone system $\frac{d z^{*}}{d t} \leq A z^{*}$ is obtained, which is a linear system and only has one equilibrium $E_{0}\{(0,0,0)\}$. Based on Lemma 1 in [45], we find that the eigenvalues of the matrix $A$ all have negative real parts when $R_{0}<1$, so the linear system is stable, and then $H_{k} \rightarrow 0, F_{k} \rightarrow 0, B_{k} \rightarrow 0$ as $t \rightarrow \infty, k=1,2,3, \ldots, n$. Using the comparison theorem in [44], it follows that $H_{k} \rightarrow 0, F_{k} \rightarrow 0, B_{k} \rightarrow 0$ as $t \rightarrow \infty, k=1,2,3, \ldots, n$, for the nonlinear system (2.5) when $R_{0}<1$. So the information-elimination equilibrium is globally asymptotically stable in $\Omega^{*}$ when $R_{0}<1$. This completes the proof.

In this section, the global attractivity of the information-prevailing equilibrium is discussed.

Theorem 3 Suppose that $\left(A_{k}(t), H_{k}(t), F_{k}(t), B_{k}(t)\right)$ is a solution of $(2.1)$, satisfying

$$
A_{k}(0)>0, \quad H_{k}(0)>0, \quad F_{k}(0)>0 \quad \text { and } \quad B_{k}(0)>0 .
$$

If $R_{0}>1$, then $\lim _{t \rightarrow \infty} \inf \left\{A_{k}(t), H_{k}(t), F_{k}(t), B_{k}(t)\right\}=\left\{A_{k}^{*}, H_{k}^{*}, F_{k}^{*}, B_{k}^{*}\right\}$, where $\left(A_{k}^{*}, H_{k}^{*}, F_{k}^{*}\right.$, $\left.B_{k}^{*}\right)$ is the unique information-prevailing equilibrium of $(2.1)$ for $k=1,2, \ldots, n$.

Proof Let us consider the Lyapunov function:

$$
\begin{aligned}
& W(t)=\left[\left(A_{k}-A_{k}^{*}\right)-\left(H_{k}-H_{k}^{*}\right)-\left(F_{k}-F_{k}^{*}\right)-\left(B_{k}-B_{k}^{*}\right)\right]^{2}, \\
& \begin{aligned}
W^{\prime}(t) & =2\left[\left(A_{k}-A_{k}^{*}\right)-\left(H_{k}-H_{k}^{*}\right)-\left(F_{k}-F_{k}^{*}\right)-\left(B_{k}-B_{k}^{*}\right)\right]\left[A_{k}^{\prime}+H_{k}^{\prime}+F_{k}^{\prime}+B_{k}^{\prime}\right] \\
& =2\left[\left(A_{k}-A_{k}^{*}\right)-\left(H_{k}-H_{k}^{*}\right)-\left(F_{k}-F_{k}^{*}\right)-\left(B_{k}-B_{k}^{*}\right)\right]
\end{aligned}
\end{aligned}
$$




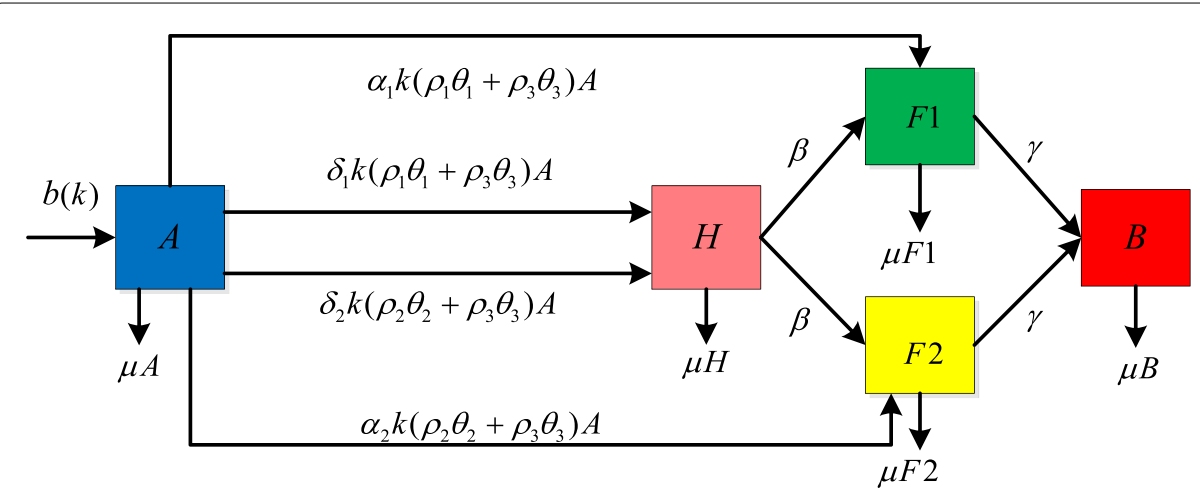

Figure 2 The flow diagram of the AHF1F2B model

$$
\times\left[b(k)-\mu A_{k}-\mu H_{k}-\mu F_{k}-\mu B_{k}\right]
$$

Because $E^{*}\left(A_{k}^{*}, H_{k}^{*}, F_{k}^{*}, B_{k}^{*}\right)$ satisfies (2.2), it follows that

$$
b(k)-\mu A_{k}^{*}-\mu H_{k}^{*}-\mu F_{k}^{*}-\mu B_{k}^{*}=0 .
$$

In other words, $b(k)=\mu A_{k}^{*}-\mu H_{k}^{*}-\mu F_{k}^{*}-\mu B_{k}^{*}$. Then, we can get

$$
\begin{aligned}
W^{\prime}(t)= & 2\left[\left(A_{k}-A_{k}^{*}\right)-\left(H_{k}-H_{k}^{*}\right)-\left(F_{k}-F_{k}^{*}\right)-\left(B_{k}-B_{k}^{*}\right)\right] \\
& \times\left[\mu A_{k}^{*}+\mu H_{k}^{*}+\mu F_{k}^{*}+\mu B_{k}^{*}-\mu A_{k}-\mu H_{k}-\mu F_{k}-\mu B_{k}\right] \\
= & 2\left[\left(A_{k}-A_{k}^{*}\right)-\left(H_{k}-H_{k}^{*}\right)-\left(F_{k}-F_{k}^{*}\right)-\left(B_{k}-B_{k}^{*}\right)\right] \\
& \times\left[\mu\left(A_{k}^{*}-A_{k}\right)-\mu\left(H_{k}^{*}-H_{k}\right)-\mu\left(F_{k}^{*}-F_{k}\right)-\mu\left(B_{k}^{*}-B_{k}\right)\right] \\
= & -2 \mu\left[\left(A_{k}-A_{k}^{*}\right)-\left(H_{k}-H_{k}^{*}\right)-\left(F_{k}-F_{k}^{*}\right)-\left(B_{k}-B_{k}^{*}\right)\right]^{2} \leq 0 .
\end{aligned}
$$

Thus, the information-prevailing equilibrium $E^{*}\left(A_{k}^{*}, H_{k}^{*}, F_{k}^{*}, B_{k}^{*}\right)$ is globally asymptotically stable by LaSalle Invariance Principle [46]. This completes the proof.

\section{A preferential information spreading model with competitive mechanism}

Competition is ubiquitous in human society $[47,48]$. Bo Zhao et al. studied the competing spreading processes of epidemic and awareness in multiplex networks, they found that strong capacities of awareness diffusion and self-protection of individuals could lead to a much higher spreading threshold and a smaller outbreak size [49]. Comparing to ecommerce networks, a product may have multiple merchants on sale, and different merchants may also offer different degrees of preferential for this product, which will trigger competition. Therefore, a preferential information spreading model with competitive mechanism is proposed. In the process of competition, we consider the difference of the preferential degree of the product and people's hesitation psychology. The model flow diagram is shown in Fig. 2.

Here $F 1_{k}(t), F 2_{k}(t)$ represent the individuals who forwarded preferential information from shop 1 and shop 2, respectively. The parameter $\beta$ represents the probability that a hesitator becomes a forwarder. A forwarder becomes a beneficiary with a probability of $\gamma$. 
The attractivity parameters $\alpha_{1}$ and $\alpha_{2}$ are used to describe the preferential degree of the product in shops 1 and 2 , respectively. The hesitation parameters $\delta_{1}$ and $\delta_{2}$ are used to describe the hesitation degree of adherents to the product preferential information in shops 1 and 2, respectively. Parameter $\rho_{1}\left(\rho_{2}\right)$ is the spread rate for an adherent after contacting with a forwarder in shop 1 (shop 2), $\rho_{3}$ is the spread rate for an adherent after contacting with a beneficiary. In this model, we assume $b(k)=\mu$. Based on these assumptions above, the model can be described by the following system of ordinary differential equations:

$$
\left\{\begin{aligned}
\frac{d A_{k}(t)}{d t}= & b(k)-\left(\alpha_{1}+\delta_{1}\right) k\left(\rho_{1} \theta_{1}(t)+\rho_{3} \theta_{3}(t)\right) A_{k}(t) \\
& -\left(\alpha_{2}+\delta_{2}\right) k\left(\rho_{2} \theta_{2}(t)+\rho_{3} \theta_{3}(t)\right) A_{k}(t)-\mu A_{k}(t), \\
\frac{d H_{k}(t)}{d t}= & \delta_{1} k\left(\rho_{1} \theta_{1}(t)+\rho_{3} \theta_{3}(t)\right) A_{k}(t) \\
& +\delta_{2} k\left(\rho_{2} \theta_{2}(t)+\rho_{3} \theta_{3}(t)\right) A_{k}(t)-2 \beta H_{k}(t)-\mu H_{k}(t), \\
\frac{d F 1_{k}(t)}{d t}= & \alpha_{1} k\left(\rho_{1} \theta_{1}(t)+\rho_{3} \theta_{3}(t)\right) A_{k}(t)+\beta H_{k}(t)-\gamma F 1_{k}(t)-\mu F 1_{k}(t), \\
\frac{d F 2_{k}(t)}{d t}= & \alpha_{2} k\left(\rho_{2} \theta_{2}(t)+\rho_{3} \theta_{3}(t)\right) A_{k}(t)+\beta H_{k}(t)-\gamma F 2_{k}(t)-\mu F 2_{k}(t), \\
\frac{d B_{k}(t)}{d t}= & \gamma F 1_{k}(t)+\gamma F 2_{k}(t)-\mu B_{k}(t) .
\end{aligned}\right.
$$

Here $\theta_{1}\left(\theta_{2}\right)$ denotes the probability that an adherent contacts with a forwarder in shop 1 (shop 2 ) at time $t, \theta_{3}$ denotes the probability that an adherent contacts with a beneficiary at time $t$, which satisfies the relation

$$
\left\{\begin{array}{l}
\theta_{1}(t)=\sum_{i=1}^{n} \frac{\varphi(i)}{i} P(i \mid k) \frac{F 1_{i}(t)}{N_{i}(t)}=\langle k\rangle^{-1} \sum_{i=1}^{n} \frac{\varphi(i)}{\eta_{i}} P(i) F 1_{i}(t), \\
\theta_{2}(t)=\sum_{i=1}^{n} \frac{\varphi(i)}{i} P(i \mid k) \frac{F 2_{i}(t)}{N_{i}(t)}=\langle k\rangle^{-1} \sum_{i=1}^{n} \frac{\varphi(i)}{\eta_{i}} P(i) F 2_{i}(t), \\
\theta_{3}(t)=\sum_{i=1}^{n} \frac{\varphi(i)}{i} P(i \mid k) \frac{B_{i}(t)}{N_{i}(t)}=\langle k\rangle^{-1} \sum_{i=1}^{n} \frac{\varphi(i)}{\eta_{i}} P(i) B_{i}(t) .
\end{array}\right.
$$

\section{Theorem 4 Consider model (5.1) and define}

$$
\begin{aligned}
R_{0}^{*}= & \frac{\langle\varphi(k) k\rangle}{\langle k\rangle} \\
& \times \frac{\left[\left(\alpha_{1}(2 \beta+\mu)+\beta \delta_{1}\right) \mu \rho_{1}+\left(\alpha_{2}(2 \beta+\mu)+\beta \delta_{2}\right) \mu \rho_{2}+\left(\left(\alpha_{1}+\alpha_{2}\right)(2 \beta+\mu)+2 \beta\left(\delta_{1}+\delta_{2}\right)\right) \gamma \rho_{3}\right]}{\mu(2 \beta+\mu)(\mu+\gamma)},
\end{aligned}
$$

then the following statements hold:

(1) There always exists an information-elimination equilibrium $E_{0}^{*}=\{(1,0,0,0,0)\}_{k}$ when $R_{0}^{*}<1$.

(2) There is an information-prevailing equilibrium $E_{1}^{*}=\left\{\left(A_{k}^{*}, H_{k}^{*}, F 1_{k}^{*}, F 2_{k}^{*}, B_{k}^{*}\right)\right\}_{k}$ when $R_{0}^{*}>1$.

Proof According to the formulas of system (5.1) and due to $b(k)=\mu$, one can easily find that $E_{0}^{*}(1,0,0,0,0)$ is always an equilibrium of system (5.1), which is called information-elimination equilibrium. To get the information-prevailing equilibrium $E_{1}^{*}=$ $\left(A^{*}, H^{*}, F 1^{*}, F 2^{*}, B^{*}\right)$, we need to make the right-hand side of system (5.1) equal to zero, 
so it should satisfy

$$
\left\{\begin{array}{l}
b-\left(\alpha_{1}+\delta_{1}\right) k\left(\rho_{1} \theta_{1}^{*}(t)+\rho_{3} \theta_{3}^{*}(t)\right) A_{k}^{*}(t) \\
\quad-\left(\alpha_{2}+\delta_{2}\right) k\left(\rho_{2} \theta_{2}^{*}(t)+\rho_{3} \theta_{3}^{*}(t)\right) A_{k}^{*}(t)-\mu A_{k}^{*}(t)=0, \\
\delta_{1} k\left(\rho_{1} \theta_{1}^{*}(t)+\rho_{3} \theta_{3}^{*}(t)\right) A_{k}^{*}(t)+\delta_{2} k\left(\rho_{2} \theta_{2}^{*}(t)+\rho_{3} \theta_{3}^{*}(t)\right) A_{k}^{*}(t) \\
\quad-2 \beta H_{k}^{*}(t)-\mu H_{k}^{*}(t)=0, \\
\alpha_{1} k\left(\rho_{1} \theta_{1}^{*}(t)+\rho_{3} \theta_{3}^{*}(t)\right) A_{k}^{*}(t)+\beta H_{k}^{*}(t)-\gamma F 1_{k}^{*}(t)-\mu F 1_{k}^{*}(t)=0, \\
\alpha_{2} k\left(\rho_{2} \theta_{2}^{*}(t)+\rho_{3} \theta_{3}^{*}(t)\right) A_{k}^{*}(t)+\beta H_{k}^{*}(t)-\gamma F 2_{k}^{*}(t)-\mu F 2_{k}^{*}(t)=0, \\
\gamma F 1_{k}^{*}(t)+\gamma F 2_{k}^{*}(t)-\mu B_{k}^{*}(t)=0,
\end{array}\right.
$$

and a direct calculation yields

$$
\begin{aligned}
A^{*} & =\frac{\mu}{(\mu+k)\left[\left(\delta_{1}+\alpha_{1}\right)\left(\rho_{1} \theta_{1}^{*}+\rho_{3} \theta_{3}^{*}\right)+\left(\delta_{2}+\alpha_{2}\right)\left(\rho_{2} \theta_{2}^{*}+\rho_{3} \theta_{3}^{*}\right)\right]} \\
H^{*} & =\frac{\mu k\left(\delta_{1}\left(\rho_{1} \theta_{1}^{*}+\rho_{3} \theta_{3}^{*}\right)+\delta_{2}\left(\rho_{2} \theta_{2}^{*}+\rho_{3} \theta_{3}^{*}\right)\right)}{(2 \beta+\mu)\left(\mu+k\left[\left(\delta_{1}+\alpha_{1}\right)\left(\rho_{1} \theta_{1}^{*}+\rho_{3} \theta_{3}^{*}\right)+\left(\delta_{2}+\alpha_{2}\right)\left(\rho_{2} \theta_{2}^{*}+\rho_{3} \theta_{3}^{*}\right)\right]\right)}, \\
F 1^{*} & =\frac{\mu k\left((2 \beta+\mu) \alpha_{1}\left(\rho_{2} \theta_{2}^{*}+\rho_{3} \theta_{3}^{*}\right)+\beta\left[\delta_{1}\left(\rho_{1} \theta_{1}^{*}+\rho_{3} \theta_{3}^{*}\right)+\delta_{2}\left(\rho_{2} \theta_{2}^{*}+\rho_{3} \theta_{3}^{*}\right)\right]\right)}{(2 \beta+\mu)\left(\mu+k\left[\left(\delta_{1}+\alpha_{1}\right)\left(\rho_{1} \theta_{1}^{*}+\rho_{3} \theta_{3}^{*}\right)+\left(\delta_{2}+\alpha_{2}\right)\left(\rho_{2} \theta_{2}^{*}+\rho_{3} \theta_{3}^{*}\right)\right]\right)} \\
F 2^{*} & =\frac{\mu k\left((2 \beta+\mu) \alpha_{2}\left(\rho_{2} \theta_{2}^{*}+\rho_{3} \theta_{3}^{*}\right)+\beta\left[\delta_{1}\left(\rho_{1} \theta_{1}^{*}+\rho_{3} \theta_{3}^{*}\right)+\delta_{2}\left(\rho_{2} \theta_{2}^{*}+\rho_{3} \theta_{3}^{*}\right)\right]\right)}{(2 \beta+\mu)\left(\mu+k\left[\left(\delta_{1}+\alpha_{1}\right)\left(\rho_{1} \theta_{1}^{*}+\rho_{3} \theta_{3}^{*}\right)+\left(\delta_{2}+\alpha_{2}\right)\left(\rho_{2} \theta_{2}^{*}+\rho_{3} \theta_{3}^{*}\right)\right]\right)}, \\
B^{*} & =\frac{\gamma \mu k\left((2 \beta+\mu)\left[\alpha_{1}\left(\rho_{1} \theta_{1}^{*}+\rho_{3} \theta_{3}^{*}\right)+\alpha_{2}\left(\rho_{2} \theta_{2}^{*}+\rho_{3} \theta_{3}^{*}\right)\right]+2 \beta\left[\delta_{1}\left(\rho_{1} \theta_{1}^{*}+\rho_{3} \theta_{3}^{*}\right)+\delta_{2}\left(\rho_{2} \theta_{2}^{*}+\rho_{3} \theta_{3}^{*}\right)\right]\right)}{\mu(\gamma+\mu)(2 \beta+\mu)\left(\mu+k\left[\left(\delta_{1}+\alpha_{1}\right)\left(\rho_{1} \theta_{1}^{*}+\rho_{3} \theta_{3}^{*}\right)+\left(\delta_{2}+\alpha_{2}\right)\left(\rho_{2} \theta_{2}^{*}+\rho_{3} \theta_{3}^{*}\right)\right]\right)},
\end{aligned}
$$

as well as $\rho(t)=\sum_{i} P(i)\left(\rho_{1} F 1_{i}+\rho_{2} F 2_{i}+\rho_{3} B_{i}\right) \leq \rho_{1}+\rho_{2}+\rho_{3}$.

Obviously, $\rho=0$ satisfies (5.3). Hence, $A_{k}=1$ and $H_{k}=F 1_{k}=F 2_{k}=B_{k}=0$ is an equilibrium of (5.1), which is called the information-elimination equilibrium.

Substituting $F 1_{k}, F 2_{k}$, and $B_{k}$ of (5.1) into $\rho$, an equation of the form $\rho f(\rho)=0$ is obtained, where

$$
\begin{aligned}
f(\rho)= & 1-\frac{\left(\alpha_{1}(2 \beta+\mu)+\beta \delta_{1}\right) \mu \rho_{1}+\left(\alpha_{2}(2 \beta+\mu)+\beta \delta_{2}\right) \mu \rho_{2}+\left((2 \beta+\mu)\left(\alpha_{1}+\alpha_{2}\right)+2 \beta\left(\delta_{1}+\delta_{2}\right)\right) \gamma \rho_{3}}{\langle k\rangle \mu(\gamma+\mu)(2 \beta+\mu)} \\
& \times \sum_{i} \varphi(k) k P(i) .
\end{aligned}
$$

Since $f^{\prime}(\rho)>0$ and $f\left(\rho_{1}+\rho_{2}+\rho_{3}\right)>0$, the equation $f(\rho)=0$ has a unique nontrivial solution if and only if $f(0)<0$, i.e., $\left[\left(\alpha_{1}(2 \beta+\mu)+\beta \delta_{1}\right) \mu \rho_{1}+\left(\alpha_{2}(2 \beta+\mu)+\beta \delta_{2}\right) \mu \rho_{2}+\gamma((2 \beta+\right.$ $\left.\left.\mu)\left(\alpha_{1}+\alpha_{2}\right)+2 \beta\left(\delta_{1}+\delta_{2}\right)\right) \rho_{3}\right]\langle\varphi(k) k\rangle /\langle k\rangle(\gamma+\mu)(2 \beta+\mu)>1$. So, we can get the basic reproductive number

$$
\begin{aligned}
R_{0}^{*}= & \frac{\langle\varphi(k) k\rangle}{\langle k\rangle} \\
& \times \frac{\left[\left(\alpha_{1}(2 \beta+\mu)+\beta \delta_{1}\right) \mu \rho_{1}+\left(\alpha_{2}(2 \beta+\mu)+\beta \delta_{2}\right) \mu \rho_{2}+\left(\left(\alpha_{1}+\alpha_{2}\right)(2 \beta+\mu)+2 \beta\left(\delta_{1}+\delta_{2}\right)\right) \gamma \rho_{3}\right]}{\mu(2 \beta+\mu)(\mu+\gamma)},
\end{aligned}
$$

hence, a nontrivial solution exists if and only if $R_{0}^{*}>1$, completing the proof. 


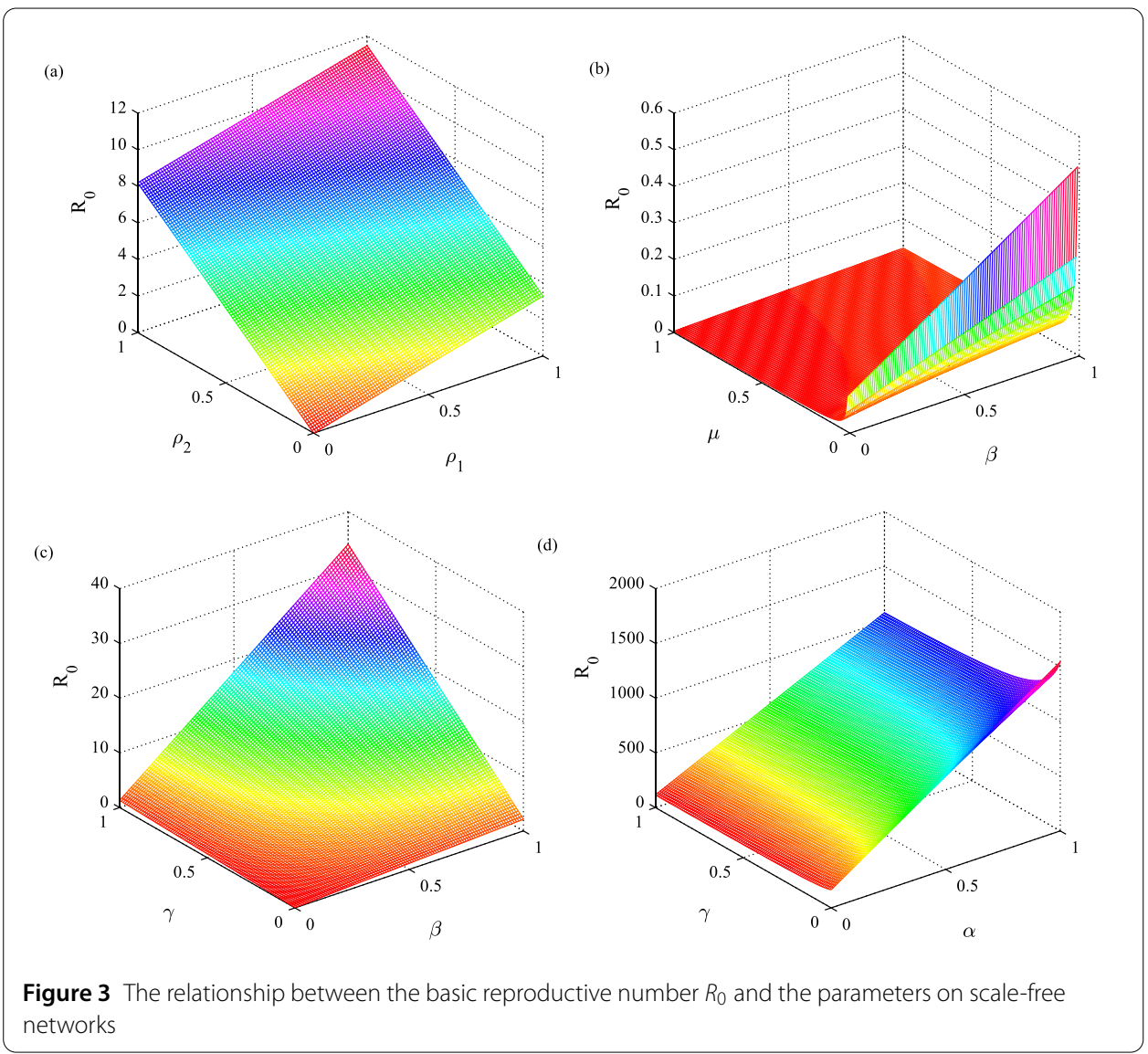

\section{Numerical simulation and discussion}

First, we perform some sensitivity analysis of the basic reproductive number $R_{0}$ in terms of model (2.1) parameters on scale-free networks. Obviously,

$$
\begin{array}{ll}
\frac{\partial R_{0}}{\partial \rho_{1}}=\frac{\langle\varphi(k) k\rangle(\alpha(\beta+\mu)+\delta \beta)}{\langle k\rangle(\beta+\mu)(\gamma+\mu)}, & \frac{\partial R_{0}}{\partial \rho_{2}}=\frac{\langle\varphi(k) k\rangle \gamma(\alpha(\beta+\mu)+\delta \beta)}{\langle k\rangle \mu(\beta+\mu)(\gamma+\mu)}, \\
\frac{\partial R_{0}}{\partial \beta}=\frac{\langle\varphi(k) k\rangle \delta\left(\mu \rho_{1}+\gamma \rho_{2}\right)}{\langle k\rangle(\gamma+\mu)(\beta+\mu)^{2}}, & \frac{\partial R_{0}}{\partial \gamma}=\frac{\langle\varphi(k) k\rangle\left(\rho_{2}-\rho_{1}\right)(\alpha(\beta+\mu)+\delta \beta)}{\langle k\rangle(\beta+\mu)(\gamma+\mu)^{2}}, \\
\frac{\partial R_{0}}{\partial \alpha}=\frac{\langle\varphi(k) k\rangle\left(\mu \rho_{1}+\gamma \rho_{2}\right)}{\langle k\rangle \mu(\gamma+\mu)}, & \frac{\partial R_{0}}{\partial \delta}=\frac{\langle\varphi(k) k\rangle \beta\left(\mu \rho_{1}+\gamma \rho_{2}\right)}{\langle k\rangle \mu(\gamma+\mu)(\beta+\mu)} .
\end{array}
$$

Some interesting results can be found as follows. In Fig. 3(a), the parameters are chosen as follows: $\beta=0.15, \gamma=0.25, \alpha=0.3, \delta=0.1, \mu=0.1$; we can see that larger $\rho_{1}$ or $\rho_{2}$ can lead to larger $R_{0}$. In Fig. 3(b), the parameters are chosen as follows: $\rho_{1}=0.3, \rho_{2}=0.25, \alpha=$ $0.35, \delta=0.4, \gamma=0.4$; it shows that larger $\beta$ can lead to larger $R_{0}$, but $R_{0}$ increase as $\mu$ decreases. In Fig. 3(c), the parameters are chosen as follows: $\rho_{1}=0.25, \rho_{2}=0.3, \beta=0.15$, which shows that larger $\gamma$ or $\delta$ can lead to larger $R_{0}$ when $\rho_{1}<\rho_{2}$. Figure 3(d) shows that larger $\alpha$ can lead to larger $R_{0}$ and larger $\gamma$ can lead to a decrease in $R_{0}$ when $\rho_{1}>\rho_{2}$. From Fig. 3(a), (c), and (d), we can see that the simultaneous effect of the two parameters has a greater impact on the basic reproductive number. At the same time, variance of degree distribution $\varphi(k) \lambda(k)$ manifests in the diversity in contact patterns. Particularly, the ratio 

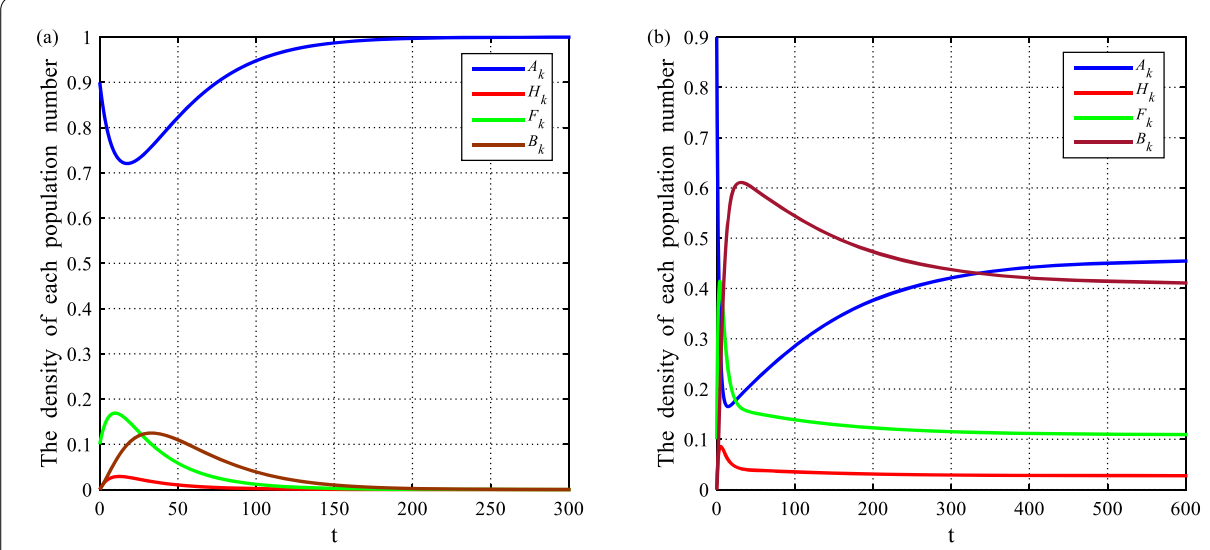

Figure 4 Population changes for each compartment over time when $R_{0}<1$ (a) and $R_{0}>1$ (b)

$\varphi(k) \lambda(k) /\langle k\rangle$ is the parameter defining the level of heterogeneity of the network, which shows the impact of network topology.

In this section, we conduct simulations to investigate the dynamics of the model parameters and network topology structures to support and explain our theoretical results. Here the degree distribution is $P(k)=c k^{-l}(2<l \leq 3)$ in which $l=3$ and $c$ satisfies $\sum_{k=1}^{n} P(k)=1$ [50], $\sum_{k=1}^{n} P(k)=1, n=4000, b(k)=b / n$; if we choose $\varphi(k)=k^{m}$ with $m=1$, then some interesting phenomena can be observed in our simulations.

Figure 4 shows the time series for $A(t), H(t), F(t)$, and $B(t)$, which are the globally average densities of the four states. The initial values of Figs. 4-8 are $A(t)=0.9, H(t)=0, F(t)=0.1$ and $B(t)=0$. In Fig. 4(a), if we choose $\rho_{1}=0.02, \rho_{2}=0.01, \delta=0.1, \mu=0.04, \beta=0.06, \gamma=$ $0.05, \alpha=0.4$, then the basic reproductive number $R_{0}=0.758<1$. It shows that, when $R_{0}<1$, the number of forwarders will diminish finally over time, even for a large fraction of the forwarder nodes at the beginning, the preferential information spreading will die out eventually, and all nodes will become adherents, i.e., $A(t)=1, H(t)=0, F(t)=0$, and $B(t)=0$ as $t \rightarrow \infty$ when $R_{0}<1$. It suggests that the information-eliminate equilibrium is locally stable when $R_{0}<1$, in agreement with Theorem 2. The parameters in Fig. 4(b) are chosen as $\rho_{1}=0.25, \rho_{2}=0.1, \alpha=0.6, \beta=0.3, \gamma=0.2, \delta=4, \mu=0.1, \alpha=0.6$, and then the basic reproductive number $R_{0}=3.07>1$. It is shown that when $R_{0}>1$, even for a small fraction of the forwarder nodes at the beginning, the density of forwarder is maintaining at a positive constant, which means that the preferential information spreading will be persistent on the network, in accordance with Theorems 3 and 4.

Figure 5 shows the dynamics behaviors of forwarders with different degree when the basic reproductive number $R_{0}<1$ and $R_{0}>1$. We find that the larger degree leads to larger value of the preferential information spreading level.

Figure 6 shows the effects of attractivity parameter $\alpha$ in $H_{200}$ and $F_{200}$. We choose the parameters as follows: $\rho_{1}=0.2, \rho_{2}=0.15, \gamma=0.2, \delta=0.15, \beta=0.1$, with $\alpha=0.1,0.4,0.7,0.9$ from bottom to top. It can be observed that the higher the attractivity rate $\alpha$, the fewer the hesitators. That is, the greater the degree of preferential, the less hesitant people will be to forward the preferential information.

Figure 7 shows the effects of parameter $\delta$ in $H_{200}$ and $F_{200}$. We choose $\rho_{1}=0.25, \rho_{2}=$ $0.1, \gamma=0.15, \alpha=0.5, \beta=0.1$, with $\delta=0.05,0.1,0.3,0.5$ from bottom to top. One can see that the higher the hesitation of the customer, the fewer individuals will be able to forward 

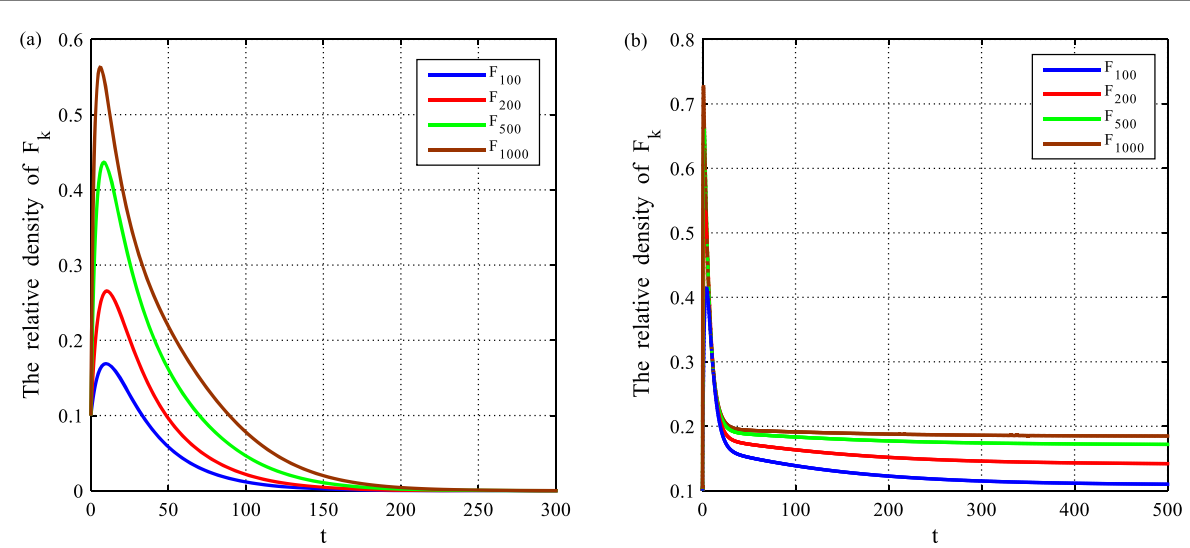

Figure 5 Dynamical behavior of forwarder with different degree when $R_{0}<1$ (a) and $R_{0}>1$ (b)
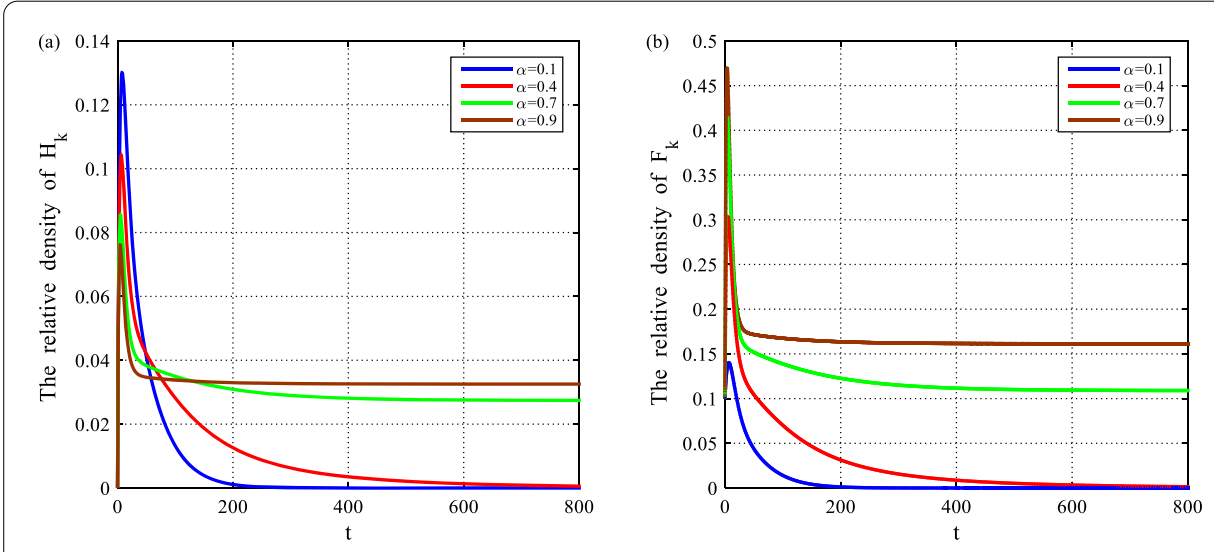

Figure 6 The density of $\mathrm{H}_{200}$ and $F_{200}$ under different $\alpha$
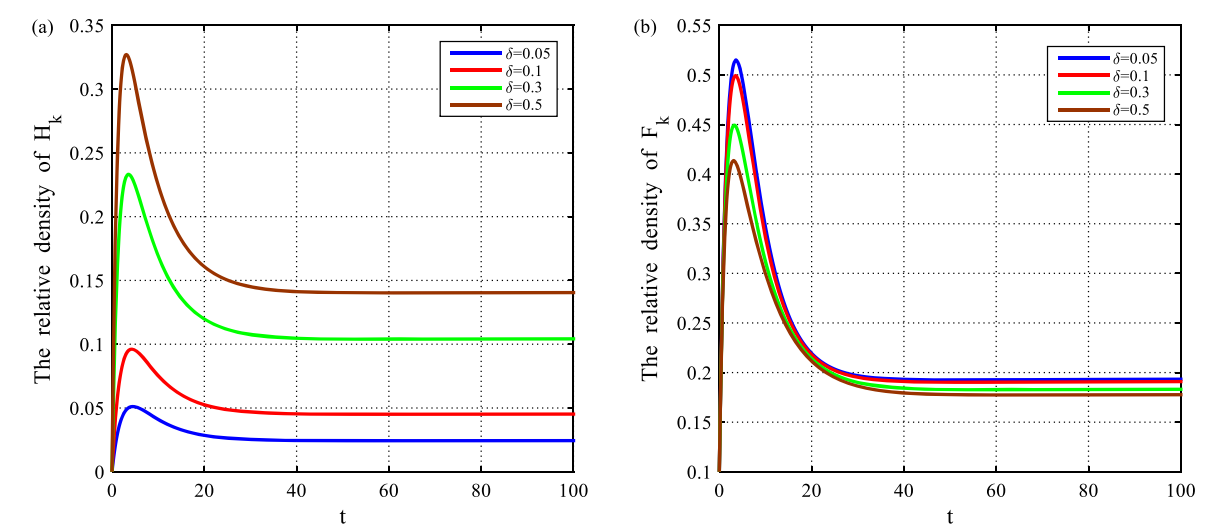

Figure 7 The density of $H_{200}$ and $F_{200}$ under different $\delta$

the preferential information. Therefore, reducing the degree of hesitation of the customer, that is, improving the promotion strategy and effective customer service guidance, can promote the spreading of the preferential information. 

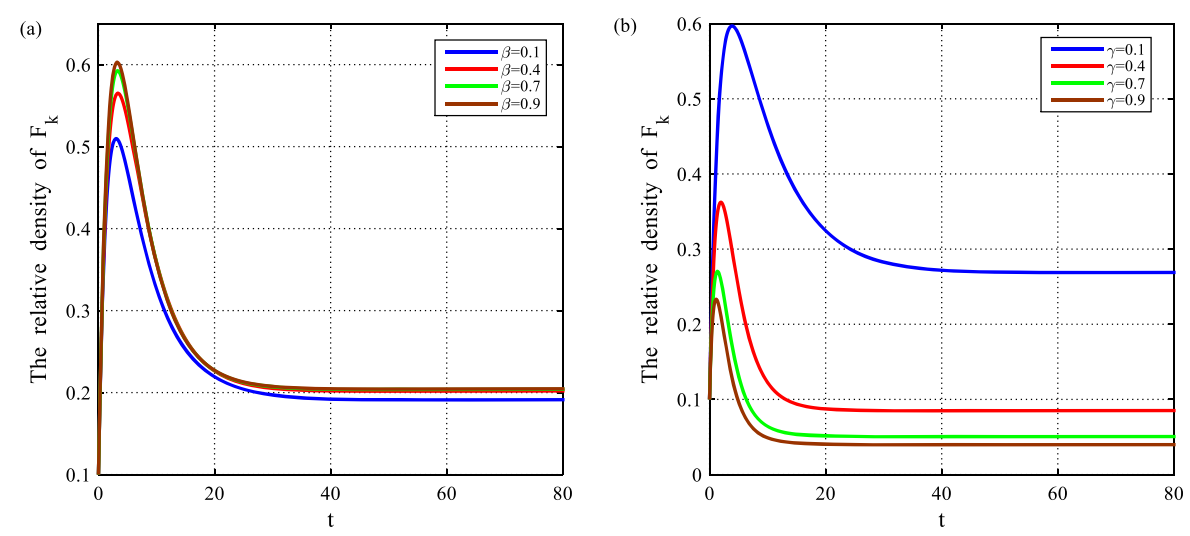

Figure 8 The density of $F_{200}$ under different $\beta$ in (a) and $\gamma$ in (b)

Figure 8 shows the effects of parameter $\beta$ and $\gamma$ in $F_{200}$. The parameters in Fig. 8(a) are chosen as follows: $\rho_{1}=0.2, \rho_{2}=0.15, \gamma=0.15, \alpha=0.6, \delta=0.2, \beta$ is chosen as 0.1, 0.4, 0.7, 0.9. This implies that the product's attractiveness parameter (the degree of preferential) plays a strong role in contributing the spreading of preferential information. In Fig. 8(b), $\beta=0.2$, the parameter $\gamma$ is chosen as $0.1,0.4,0.7,0.9$. It can be seen that if it is easier to get preferential, the number of beneficiaries will increase.

Figure 9 compares the density of susceptible (adherent), sharer, beneficiary in the SIB and $A H F B$ models. In Fig. 9(a), we choose parameters $\mu=0.2, \beta=0.2, \alpha=0.4, \rho_{1}=$ $0.1, \rho_{2}=0.01, R_{0}=0.5008$ in AHFB model. In Fig. 9(b) and (c), we choose $\mu=0.1, \beta=$ $0.3, \alpha=0.7, \rho_{1}=0.5, \rho_{2}=0.01, R_{0}=4.223$. It can be easily seen that the peak value of each state' density of SIB model is higher than that of $A H F B$ model. It illustrates that the hesitation mechanism can reduce the level of preferential information spreading, but it can reflect people's identification of some preferential information and people's rational shopping during shopping.

Figure 10 shows the time series for $A(t), H(t), F 1(t), F 2(t)$, and $B(t)$ in preferential information competition model, which are the globally average densities of the five states. The initial value of Fig. 10 is $A(t)=0.8, H(t)=0, F 1(t)=0.1, F 2(t)=0.1$, and $B(t)=0$. In Fig. 10(a), we choose the parameters as $\mu=0.04, \rho_{1}=0.05, \rho_{2}=0.05, \rho_{3}=0.01, \delta_{1}=$ $0.15, \delta_{2}=0.1, \beta=0.15, \gamma=0.25, \alpha_{1}=0.4, \alpha_{2}=0.3$, forwarders 1 and 2 will disappear finally over time. And in Fig. 10(b), the parameters are chosen as $\rho_{1}=0.08, \rho_{2}=0.08, \rho_{3}=$ $0.05, \delta_{1}=0.15, \delta_{2}=0.2, \beta=0.24, \gamma=0.22, \alpha_{1}=0.5, \alpha_{2}=0.4$. We can see that the population of forwarders will be maintained at a positive constant, which means that the information spreading will persistent.

Figure 11 further studies the impact of preferential levels on competition mechanisms. In Figs. 11(a), if the merchant's preferential strength is less $\left(0<\alpha_{1}, \alpha_{2} \leq 0.1\right)$, we choose $\alpha_{2}=0.03, \alpha_{1}=0.031, \ldots, 0.039,0.04$. It can see that in the process of competition, when the degree of merchandise preference is 0.001 more than that of competitors, a larger number of forwarders can be obtained, when the preference is increased again, the degree of increase is smaller. In Fig. 11(b), if the merchant's preferential strength is large $\left(0.1<\alpha_{1}, \alpha_{2}<1\right)$, we choose $\alpha_{2}=0.5, \alpha_{1}=0.51, \ldots, 0.59,0.6$. We can see that in the competition process, when the degree of merchandise preference is 0.01 higher than that of the competitor, the number of forwarders will increase significantly, and when the preference 

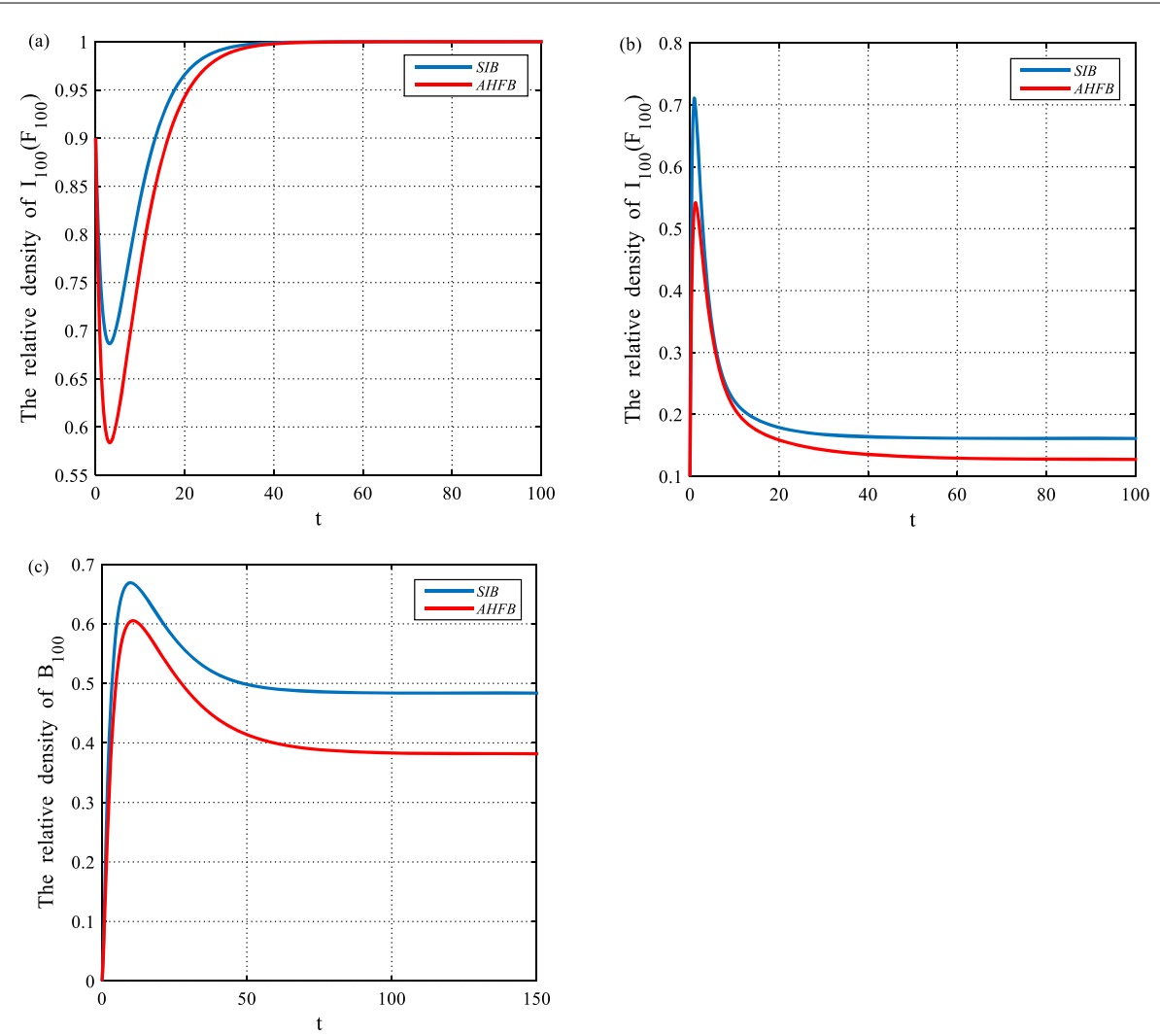

Figure 9 Population changes for each compartment over time in SIB and SHFB models
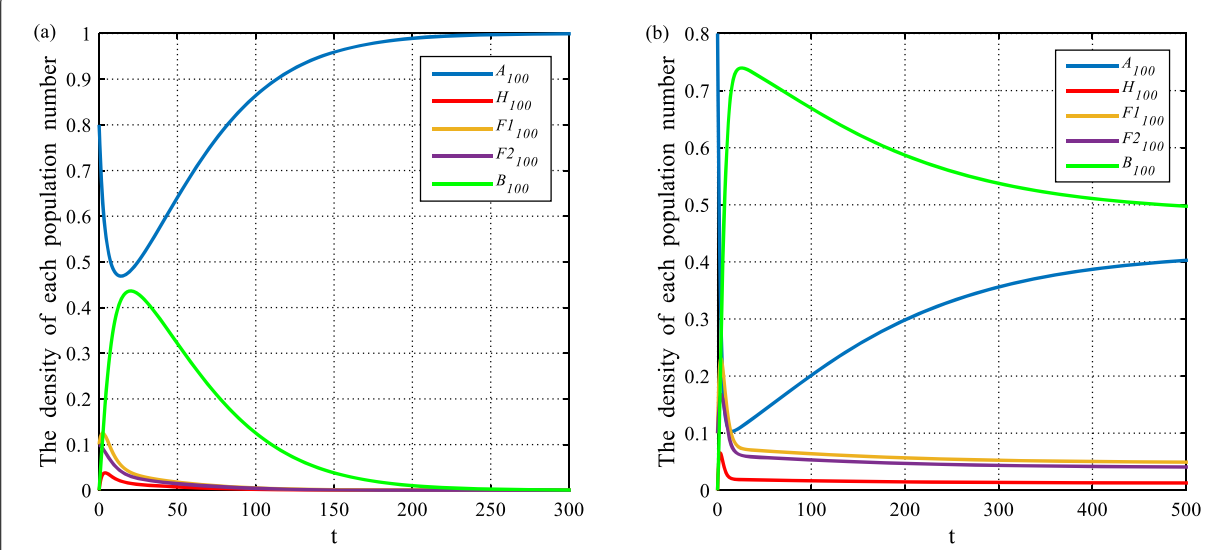

Figure 10 Population changes for each compartment over time in competition model when $R_{0}<1(\mathbf{a})$ and $R_{0}>1$ (b)

is increased again, the degree of increase is not obvious. From this we can find the best preferential policies in the competition process.

Figure 12 shows the impact of beneficiary spreading rate on hesitators and forwarders. The parameters are chosen as $\rho_{1}=0.2, \rho_{2}=0.2, \delta_{1}=0.15, \delta_{2}=0.15, \beta=0.24, \gamma_{1}=0.25, \alpha_{1}=$ $0.5, \alpha_{2}=0.5$, and $\rho_{3}$ is chosen as $0.05,0.1,0.2$. It shows that the beneficiary spreading rate has little effect on the hesitators, but it has a great influence on the forwarders. Hence, 

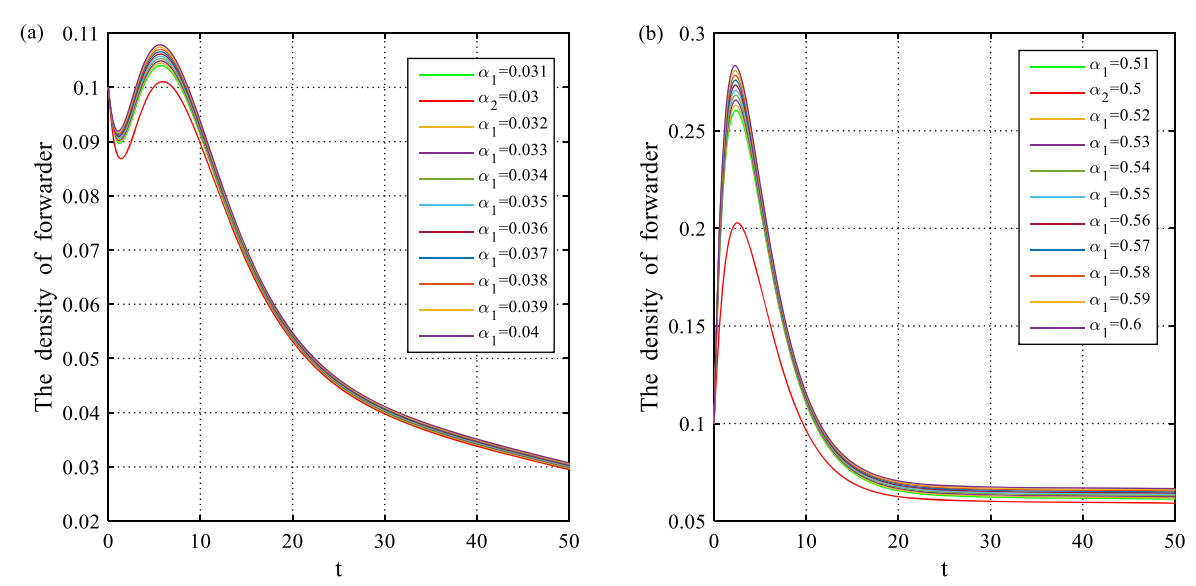

Figure 11 The density of $F 1$ and $F 2$ under different $\alpha_{1}, \alpha_{2}$
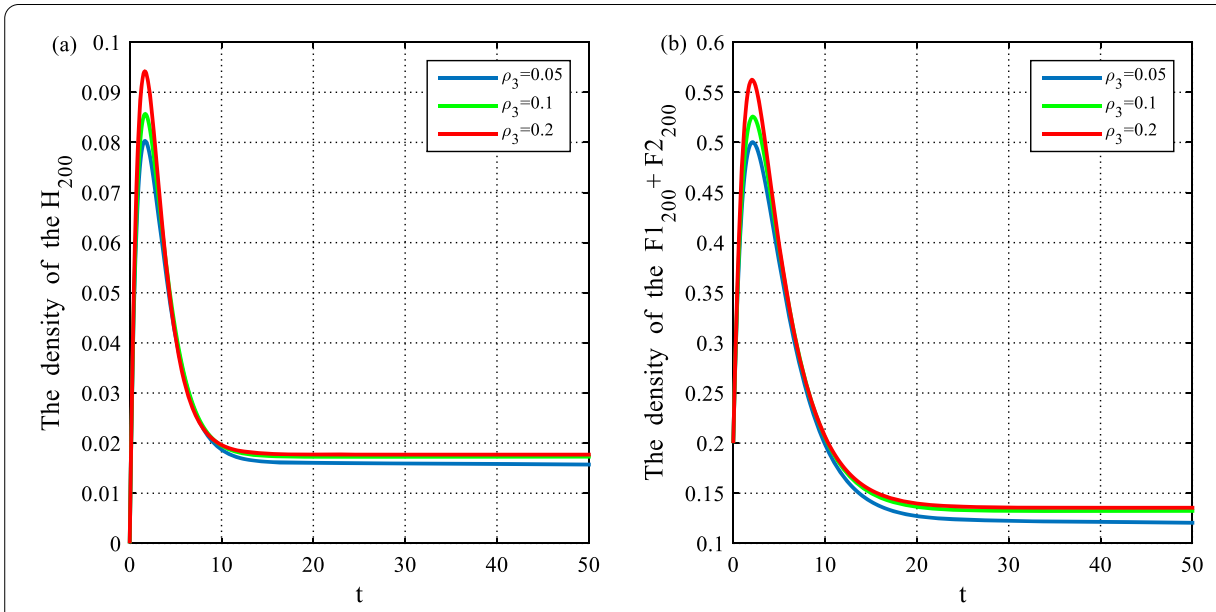

Figure 12 The density of $\mathrm{H}_{200}$ and $F 1_{200}+F 2_{200}$ under different $\rho_{3}$

increasing the acknowledged comment and positive feedback of the beneficiaries can help to increase the number of forwarders.

\section{Conclusion}

In this paper, we propose a novel $A H F B$ model to describe the dynamic behavior of preferential information spreading on scale-free networks. We obtain the basic reproductive number $R_{0}$ with the next generation matrix method, which is closely related to the topology of the underlying networks and some model parameters. Interestingly, the basic reproductive number $R_{0}$ has no relationship with the degree-dependent new register $b(k)$. More specifically, by using the comparison principle and Lyapunov function, we prove that the information-eliminate equilibrium $E_{0}$ is globally asymptotically stable when $R_{0}<1$; the preferential information is uniformly persistent on the network when $R_{0}>1$. In all cases, the effects of attractiveness in preferential information and the hesitation psychology of customers on the information spreading dynamics have been discussed. It seems that increasing the attractiveness parameter $\alpha$ or decreasing the hesitation parameter $\delta$ 
can improve preferential information spreading. Moreover, we study the preferential information spreading model with competitive mechanism and analyze corresponding dynamics behavior, then we draw a conclusion that the degree of preferential for products in the process of commodity competition is a core competitiveness factor. We have found that the degree of merchandise preference is 0.001 or 0.01 more than that of competitors and the number of forwarders will increase significantly. At the same time, increasing the spreading rate of beneficiaries, that is, the acknowledged comment and positive feedback of the beneficiaries can help promote the sale of products. The study has valuable guiding significance in effectively managing and controlling preferential information spreading on scale-free networks.

\section{Acknowledgements}

We thank the referees and the editor for their careful reading of the original manuscript and many valuable comments and suggestions that greatly improved the presentation of this paper.

\section{Funding}

This work is supported by the National Natural Science Foundation of China under Grant 61672112, 61873287, Project in Hubei Province Department of Education under Grant B2016036 and Yangtze University Excellent Doctoral and Master's Thesis Cultivation Program Funding Project.

\section{Competing interests}

The authors declare that they have no competing interests

\section{Authors' contributions}

The authors contributed equally to this work. All authors read and approved the final manuscript.

\section{Author details}

${ }^{1}$ National Demonstration Center for Experimental Electrical and Electronic Education, Yangtze University, Jingzhou, P.R. China. ${ }^{2}$ School of Electronics and Information, Yangtze University, Jingzhou, P.R. China. ${ }^{3}$ College of Information Science and Engineering, Central South University, Changsha, P.R. China.

\section{Publisher's Note}

Springer Nature remains neutral with regard to jurisdictional claims in published maps and institutional affiliations.

Received: 26 January 2019 Accepted: 27 June 2019 Published online: 09 July 2019

\section{References}

1. Zeng, Z.Y., Rui Shen, L.I: A survey of the research on propagation model of network public opinion. J. China Acad. Electron. Inf. Technol. 11(6), 588-593 (2016)

2. Wang, Z., Zhao, H., Lai, Z., et al.: Improved SIR epidemic model of social network marketing effectiveness and experimental simulation. Syst. Eng. 36(8), 2024-2034 (2016)

3. Meng, Q., Zhang, N., Zhao, X., et al.: The governance strategies for public emergencies on social media and their effects: a case study based on the microblog data. Electron. Mark. 26(1), 15-29 (2016)

4. Wu, W.W.: The cooperation-competition model for the hot topics of Chinese micro-blogs. Appl. Mech. Mater. 380 2724-2727 (2013)

5. Barabási, A.L.: The network takeover. Nat. Phys. 8, 14 (2011)

6. Lee, J.S., Lin, K.S.: Electron. Commer. Res. Appl. 12, 1-13 (2013)

7. Cordasco, G., Gargano, L., Rescigno, A.A.: Active influence spreading in social networks. Theor. Comput. Sci. 764, $15-29$ (2019)

8. Li, C.: A study on time-delay rumor propagation model with saturated control function. Adv. Differ. Equ. 1, 255 (2017)

9. Zhan, X.S., Guan, Z.H., Zhang, X.H., et al.: Optimal tracking performance and design of networked control systems with packet dropouts. J. Franklin Inst. 350(10), 3205-3216 (2013)

10. Zan, Y.: DSIR double-rumors spreading model in complex networks. Chaos Solitons Fractals 110, 191-202 (2018)

11. Lin, T., Fan, C., Liu, C., et al.: Optimal control of a rumor propagation model with latent period in emergency event. Adv. Differ. Equ. 1, 54 (2015)

12. Zhang, Y., Wang, Z., Zou, L., Fang, H.: Event-based finite-time filtering for multi-rate systems with fading measurements. IEEE Trans. Aerosp. Electron. Syst. 53(3), 1431-1441 (2017)

13. Liu, X., Li, T., Xu, H., et al.: Spreading dynamics of an online social information model on scale-free networks. Physica $A$ $514,497-510(2019)$

14. Zhan, X.X., Liu, C., Zhou, G., et al.: Coupling dynamics of epidemic spreading and information diffusion on complex networks. Appl. Math. Comput. 332, 437-448 (2018)

15. Zhan, X.S., Guan, Z.H., et al.: Best tracking performance of networked control systems based on communication constraints. Asian J. Control 16(4), 1155-1163 (2014)

16. Li, T., Liu, X., Wu, J., et al.: An epidemic spreading model on adaptive scale-free networks with feedback mechanism. Physica A 450, 649-656 (2016) 
17. Zhan, X.S., Wu, J., Jiang, T., et al.: Optimal performance of networked control systems under the packet dropouts and channel noise. ISA Trans. 58, 214-221 (2015)

18. Jiang, X.W., Ding, L., Guan, Z.H., et al.: Bifurcation and chaotic behavior of a discrete-time Ricardo-Malthus model. Nonlinear Dyn. 71(3), 437-446 (2013)

19. Zhu, X., Wang, W., Cai, S., et al.: Dynamics of social contagions with local trend imitation. Sci. Rep. 8(1), 7335 (2018)

20. Zhang, L., Zhu, L., Yang, Y.: Describing the information spreading on a small-world network based on state space. Proc. Comput. Sci. 107, 91-96 (2017)

21. Lim, S., Shin, J., Kwak, N., et al.: Phase transitions for information diffusion in random clustered networks. Eur. Phys. J. B 89(9), 188-203 (2016)

22. Liu, X., He, D., Yang, L., et al.: A novel negative feedback information dissemination model based on online social network. Physica A 513, 371-389 (2019)

23. Du, J., Jiang, C., Chen, K.C., et al.: Community-structured evolutionary game for privacy protection in social networks. IEEE Trans. Inf. Forensics Secur. 13(3), 574-589 (2018)

24. Kanavos, A., Perikos, I., Hatzilygeroudis, I., et al.: Emotional community detection in social networks. Comput. Electr. Eng. 65, 449-460 (2018)

25. Zhang, Y., Wang, Z., Alsaadi, F.E.: Detection of intermittent faults for nonuniformly sampled multirate systems with dynamic quantization and missing measurements. Int. J. Control 1-23 (2018)

26. Zhang, Y., Wang, Z., Ma, L.: Variance-constrained state estimation for networked multi-rate systems with measurement quantization and probabilistic sensor failures. Int. J. Robust Nonlinear Control 26(16), 3507-3523 (2016)

27. Zhai, X., Zhou, W., Fei, G., et al.: Null model and community structure in multiplex networks. Sci. Rep. 8(1), 3245 (2018)

28. Kan, J.Q., Zhang, H.F.: Effects of awareness diffusion and self-initiated awareness behavior on epidemic spreading-an approach based on multiplex networks. Commun. Nonlinear Sci. Numer. Simul. 44, 193-203 (2017)

29. Du, J., Jiang, C., Chen, K.C., et al.: Community-structured evolutionary game for privacy protection in social networks, IEEE Trans. Inf. Forensics Secur. 13(3), 574-589 (2018)

30. Zhu, P., Wang, X., Zhi, Q., et al.: Analysis of epidemic spreading process in multi-communities. Chaos Solitons Fractals $109,231-237(2018)$

31. Kanavos, A., Perikos, I., Hatzilygeroudis, I., et al.: Emotional community detection in social networks. Comput. Electr. Eng. 65, 449-460 (2018)

32. Ally, A.F., Zhang, N.: Effects of rewiring strategies on information spreading in complex dynamic networks. Commun. Nonlinear Sci. Numer. Simul. 57, 97-110 (2018)

33. Lin, T., Fan, C., Liu, C., et al.: Optimal control of a rumor propagation model with latent period in emergency event. Adv. Differ. Equ. 1, 54 (2015)

34. Liu, X., Li, T., Tian, M.: Rumor spreading of a SEIR model in complex social networks with hesitating mechanism. Adv. Differ. Equ. 1, 391 (2018)

35. Huang, S., Chen, F., Chen, L.: Global dynamics of a network-based SIQRS epidemic model with demographics and vaccination. Commun. Nonlinear Sci. Numer. Simul. 43, 296-310 (2017)

36. Li, T., Wang, Y., Guan, Z.H.: Spreading dynamics of a SIQRS epidemic model on scale-free networks. Commun. Nonlinear Sci. Numer. Simul. 19, 686-692 (2014)

37. Trpevski, D., Tang, W.K.S., Kocarev, L.: Model for rumor spreading over networks. Phys. Rev. E 81(5), 056102 (2010)

38. Huo, L., Wang, L., Song, N., et al.: Rumor spreading model considering the activity of spreaders in the homogeneous network. Physica A 468, 855-865 (2017)

39. Liu, W., Li, T., Liu, X., et al.: Spreading dynamics of a word-of-mouth model on scale-free networks. IEEE Access 6 , 65563-65572 (2018)

40. Jiang, X.W., Zhan, X.S., Jiang, B.: Stability and Neimark-Sacker bifurcation analysis for a discrete single genetic negative feedback autoregulatory system with delay. Nonlinear Dyn. 76(2), 1031-1039 (2014)

41. Xu, H., Li, T., Liu, X., Dong, J.: Spreading dynamics of an online social rumor model with psychological factors on scale-free networks. Physica A 252, 234-246 (2019)

42. Fu, M., Yang, H., Feng, J., et al.: Preferential information dynamics model for online social networks. Physica A 506, 993-1005 (2018)

43. Wan, C., Li, T., Guan, Z.H., et al.: Spreading dynamics of an e-commerce preferential information model on scale-free networks. Physica A 467, 192-200 (2017)

44. van den Driessche, P., Watmough, J.: Reproduction numbers and sub-threshold endemic equilibria for compartmental models of disease transmission. Math. Biosci. 180, 29-48 (2002)

45. Glavas, M., Weinberg, J.: Stability Analysis of Nonlinear Systems. Marcel Dekker, New York (1989)

46. Lasalle, J.P.: The Stability of Dynamical Systems. SIAM, Philadelphia (1976)

47. Mišić, B., Betzel, R.F., Nematzadeh, A., et al.: Cooperative and competitive spreading dynamics on the human connectome. Neuron 86(6), 1518-1529 (2015)

48. Xie, X., Xue, Y., Wu, R.: Global attractivity of a discrete competition model of plankton allelopathy with infinite deviating arguments. Adv. Differ. Equ. 1, 303 (2016)

49. Yu, S.: Extinction for a discrete competition system with feedback controls. Adv. Differ. Equ. 1, 9 (2017)

50. Zhu, G.H., Fu, X.C., Chen, G.R.: Spreading dynamics and global stability of a generalized epidemic model on complex heterogeneous networks. Appl. Math. Model. 36, 5808-5817 (2012) 